\title{
Serotipo, Patrón de Infección y Dengue Hemorrágico en Área Endémica Colombiana
}

\section{Dengue hemorrhagic fever serotype and infection pattern in a Colombian endemic area}

Raquel E. Ocazionez, Sergio Y. Gómez y Fabián M. Cortés.

Centro de Investigaciones en Enfermedades Tropicales, Universidad Industrial de Santander. Bucaramanga, Colombia. relocaz@uis.edu.co, sergioyebrail@hotmail.com,

Recibido 28 Julio 2006/Enviado para Modificación 20 Marzo 2007/Aceptado 2 Mayo 2007

\section{RESUMEN}

Objetivo La relación entre serotipo del virus, patrón de infección y dengue hemorrágico es presentada.

Métodos Se estudiaron 1545 pacientes febriles de municipios del Departamento de Santander, Colombia, entre 1998-2004. El dengue se confirmó por ELISA-IgM y el aislamiento viral se hizo en células C6/36. El patrón de infección se estableció investigando anticuerpos IgG en suero agudo. El título de anticuerpos neutralizantes se determinó usando la prueba de neutralización por reducción de placa (PRNT). Resultados Predominancia del DEN-1 en 1998 y re-introducción del DEN-3 en 2001 coincidieron con epidemias. El dengue hemorrágico fue más frecuente en infecciones por virus DEN-2 que DEN-3 (24,5\% vs 11,2 \%; $p<0,05)$. El DEN-2 se asoció más con infección secundaria que el DEN-3 (56,8 \% vs 15,7 \%; $p<0,001)$. Disminución anual del DH correlacionó con disminución de la dominancia del DEN-2 $(r=0,95, p=0,01)$ y de la infección secundaria $(r=0,9 ; p=0,03)$ e incremento de la dominancia del DEN-3 $(r=-0,91 ; p=0,03)$. No se encontraron diferencias en el título de anticuerpos neutralizantes en los casos analizados. Los anticuerpos neutralizantes del DEN-1 fueron los de mayor título.

Conclusión Cambios en la abundancia relativa de serotipos del virus se asociaron con cambios en el patrón de infección y frecuencia del dengue hemorrágico. La vigilancia virológica permanente deberá ser prioridad para la prevención del dengue hemorrágico en áreas endémicas.

Palabras Clave: dengue hemorrágico, epidemia, virus del dengue, serotipificación (fuente: DeCS,BIREME).

\section{ABSTRACT}

Objective Describing the relationship between viral serotypes, infection pattern and dengue hemorrhagic fever. 
Methods 1545 febrile patients were studied from 1998-2004 in the Santander department of Colombia. Dengue infection was confirmed by IgM ELISA and the virus was isolated in C6/36 cells. Infection pattern was established by detecting IgG antibodies in acute serum. Neutralising antibody titres were investigated in dengue cases occurring during years when less (1998) and more (2001) dengue hemorrhagic cases were reported by using PRNT.

Results DEN-1 predominance in 1998 and the re-introduction of DEN-3 in 2001 coincided with an epidemic. DEN-2 infection caused more hemorrhagic cases than DEN-3 infection $(24,5 \%$ cf $11,2 \%$; $p<0.05)$. DEN-2 was more associated with secondary infection than DEN-3 (56,8 \% cf 15,7 \%; $p<0.001)$. An annual decrease of DHF was correlated with decreased DEN-2 dominance $(r=0.95 ; p=0.01)$, and secondary infection $(r=0.9 ; p=0.03)$ and increased DEN-3 predominance $(r=-0.91$; $\mathrm{p}=0.03$ ). There were no differences in neutralising antibody titres amongst analysed cases. DEN-1 neutralising antibodies presented the highest titres.

Conclusions Change in relative dengue virus serotype abundance was associated with changed infection pattern and DHF frequency. Continuing virological surveillance should become a priority for preventing dengue hemorrhagic fever in endemic areas.

Key Words: Dengue, DEN-2, DEN-3, epidemic (source: MeSH, NLM).

$\mathrm{E}$ xisten cuatro serotipos del virus del dengue (DEN-1,2,3,4) y cada uno corresponde a una población de cepas agrupadas en varios genotipos (14). Los virus se transmiten al hombre a través de picadura de mosquitos del género Aedes y la infección puede resultar asintomática o en un síndrome febril de severidad variada. La mayoría de los enfermos desarrollan la forma leve o dengue clásico (DC) y algunos la hemorrágica que puede llevar a la muerte cuando se acompaña del síndrome de choque hipovolémico (DH/SCH). Los individuos que con mayor frecuencia sufren $\mathrm{DH} / \mathrm{SCH}$ han tenido una infección anterior por un serotipo diferente del virus $(5,6)$. Numerosas evidencias demuestran que los anticuerpos de la infección primaria se unen al virus de la secundaria formándo un complejo que es fagocitado por el macrófago a través del receptor Fcy. Como consecuencia más células resultan infectadas liberándose mayores niveles de citoquinas y mediadores químicos que incrementan la permeabilidad vascular $(7,8)$. No obstante, casos de DH/SCH ocurren por infección primaria y esto depende principalmente del serotipo. Los virus DEN-2 y DEN-3 son los que más casos severos causan seguidos por el DEN-1 y DEN-4 $(5,9)$.

En América, casos de DH fueron infrecuentes en la década de los 70 y esto se asoció a la circulación de cepas no virulentas y de un solo serotipo en la mayoría de los países $(10,11)$. En 1981 y 1994 se introdujeron las cepas de 
DEN-2 genotipo III y DEN-3 genotipo III, respectivamente, las cuales venían ocasionando la mayoría de casos hemorrágicos en países asiáticos. La primera causó la primera epidemia de DH en América ocurrida en Cuba con 10312 casos y 158 muertes y la segunda se asoció con más casos severos en Panamá y Nicaragua $(12,13)$. El incremento del turismo entre países del Continente y las deficiencias de los programas de control del Aedes aegypti en la mayoría, han promovido el desplazamiento geográfico de los virus resultando en la diseminación de las cepas virulentas y la circulación simultánea de varios serotipos en un mismo país favoreciendo las infecciones secundarias. Como consecuencia, se ha visto un incremento notorio del DH en América en los últimos 20 años: de un total de 492820 casos con 77000 hemorrágicos en la década de los 90 a 1015420 con 14374 severos solamente en el año 2002 (14).

En Colombia, la circulación de virus del dengue se detectó por primera vez en los años 70 cuando ocurrieron epidemias por DEN-1, DEN-2 y DEN-3 y más tarde en 1986 cuando el DEN-4 apareció (16). El número de casos se ha incrementado cada año en la última década, entre 2004-2006 un total de 26 331 casos confirmados por laboratorio fueron registrados por la Organización Panamericana de la Salud (17). En el Departamento de Santander el dengue es endémico con epidemias cada 2-3 años, entre 1998 y 2004 la Secretaría de Salud Departamental informó 45823 casos de los cuales 4634 se consideraron hemorrágicos (18).

Dado que no existe tratamiento antiviral ni vacuna, la prevención de epidemias de DH se basa en el monitoreo y control permanente de factores que contribuyen a la transmisión y severidad (19). Este reporte presenta la distribución anual de serotipos del virus, el patrón de infección (primaria vs secundaria) y la respuesta de anticuerpos neutralizantes asociados a la frecuencia del DH en el Departamento de Santander en el período 1998-2004.

\section{METODOLOGÍA}

Casos

Se incluyeron pacientes febriles con sospecha clínica de dengue que fueron atendidos en instituciones de salud de cuatros municipios del departamento de Santander (B/manga, Floridablanca, Girón y Piedecuesta). Entre el 22 de febrero de 1998 y 26 de diciembre de 2004, cada semana de todos los meses del período, fueron incluidos un total de 1545 casos ( 221/mes). Los de 1998 - 2002 fueron seleccionados por los médicos de cada institución de salud 
y reportados al sistema de vigilancia de enfermedades (SIVIGILA) de la Secretaría de Salud Departamental (SSD). Los incluidos entre 2003-2004 fueron seleccionados para un estudio clínico realizado por investigadores del Centro de Investigaciones Epidemiológicas (UIS). Del primer grupo se obtuvo copia autorizada de la ficha de reporte donde se registró información demográfica, clínica y pruebas de laboratorio.

\section{Sueros}

De cada caso se obtuvo una muestra de los primeros cinco días de síntomas (agudo) y en algunos otra 8-10 días después. Los sueros de 1998-2002 se seleccionaron de los remitidos al Laboratorio de Salud Pública (LSP) de la SSD y las restantes fueron enviados directamente al Laboratorio de Arbovirus del CINTROP.

Diagnóstico del dengue

Se buscaron anticuerpos séricos IgM usando el ELISA de captura. En casos del SIVIGILA los sueros se procesaron en el LSP-SSD por el UMELISA (IPK. La Habana, Cuba) y el resultado se consultó en el libro de registro previa autorización. En los otros, la prueba se hizo en el Laboratorio de Arbovirus siguiendo el procedimiento previamente descrito (20). Todo paciente con anticuerpos IgM se consideró caso de dengue laboratorio-positivo.

Patrón de infección

Se investigó en 596 casos laboratorio-positivo incluidos en 1998 ( $\mathrm{n}=80), 2000$ $(\mathrm{n}=84), 2001(\mathrm{n}=128), 2002(\mathrm{n}=106), 2003(\mathrm{n}=80)$ y $2004(\mathrm{n}=118)$. El promedio de edad (años) de los pacientes de cada año fue $25 \pm 19$; 22 \pm 18 ; 24 \pm 17 ; $24 \pm 18$; $27 \pm 17 ; 26 \pm 17$, respectivamente. La infección primaria o secundaria se confirmó por ausencia o presencia de anticuerpos IgG en el suero agudo, respectivamente.

Frecuencia del dengue hemorrágico

Se consideró como: a) porcentaje de casos hemorrágicos documentado por la SSD (18) y b) frecuencia del DH en casos de quienes el virus se aisló y para esto incluyeron 39 pacientes del 2005 - 2006. La severidad de los casos del SIVIGILA se estableció consultando la ficha de reporte y la de los otros los médicos que hicieron el seguimiento suministraron personalmente la información. Un caso de DH fue quien presentó simultáneamente fiebre, trombocitopenia (plaquetas $\leq 100000 / \mathrm{mm}^{3}$ ), algún tipo de hemorragia y evidencia de incremento de la permeabilidad vascular (cambio $\geq 20 \%$ del hematocrito en por lo menos tres valoraciones acompañado o no de derrame pleural o ascitis. 
Pruebas de Laboratorio

- $\quad$ Aislamiento viral

Se usaron células de mosquito Aedes albopictus (clon C6/36) siguiendo el procedimiento previamente descrito (20). Brevemente, cultivos celulares infectados con el suero se centrifugaron por $30 \mathrm{~min}$ a $1000 \mathrm{x}$ g, 28o C. y después de la adición de $1 \mathrm{ml}$ de medio fueron incubados a 32oC por 12 días. El aislamiento del virus se confirmó por IFD con anticuerpo policlonal (M29341; CDC, Puerto Rico) y el serotipo por la misma técnica usando anticuerpos monoclonales (CDC, Puerto Rico).

- $\quad$ ELISA-IgG

La presencia de anticuerpos IgG anti-dengue en el suero agudo se determinó usando un protocolo modificado del ELISA de captura previamente descrito (20). Brevemente, el suero se adicionó a microplacas cubiertas con anti-IgG humana por 2 hs a $37^{\circ} \mathrm{C}$, luego de 5 lavados se agregó el antígeno (CDC, Puerto Rico) por $18 \mathrm{~h}$ a $4^{\circ} \mathrm{C}$. y finalmente el conjugado (CDC, Puerto Rico) por $1 \mathrm{~h} \mathrm{a} 37^{\circ} \mathrm{C}$. Enseguida de adicionar el sustrato la intensidad del color se midió por espectofotometría. El resultado se consideró positivo cuando el valor de la DO fue 2DS del control negativo.

- $\quad$ Prueba de neutralización por reducción de placa (PRNT)

Se usó una modificación del protocolo usado por Vorndam \& Beltrán (21). Brevemente, suero inactivado fue incubado con virus por $2 \mathrm{~h}$ a temperatura ambiente, luego la mezcla se adicionó a células Vero crecidas en placas de 24 pozos y los cultivos se incubaron a 37oC $\left(5 \% \mathrm{CO}_{2}\right)$ por 5 días después de agregar medio MEM /agar $2 \%$ (GIBCO, BRL). Las placas de infección se visualizaron por coloración con rojo neutro (3,2 \%) y la reducción del número $\geq 85 \%$ con respecto al control confirmó la presencia de anticuerpos. Se hicieron titulaciones de punto final y el título fue la dilución mayor del suero a la que se detectó reducción de placas.

Análisis de los datos

La frecuencia de DH, infección secundaria y dominancia de serotipo se compararon usando regresión lineal. La frecuencia de DH e infección por DEN-2 y DEN-3 se comparó aplicando la prueba del Chi cuadrado y los títulos de anticuerpos neutralizantes se compararon con la prueba de Mann-Whitney $U$. Se utilizó el paquete SPSS para Windows versión 13.0 y valores de $P \leq$ 0,05 se consideraron significantes. 


\section{RESULTADOS}

Serotipos del virus dengue

La tasa de aislamiento viral (número de aislados/total de sueros IgM+ por 100) varió de acuerdo con el año. En 1998 fue 13,5 \% (14/104) y luego disminuyó a $5,9 \%(4 / 67)$ en 1999, pero a partir de este año se incrementó de 9,2 \% (10/ 109) en el 2000 a 24,7 \% en el 2003 (38/154). Las mayores tasas se observaron en los años con epidemias, 1998 y 2001 (18). Se identificaron los cuatro serotipos en un total de 129 virus aislados, el DEN-3 fue el más frecuente $(58,2 \%)$ seguido por el DEN-2 (22,8\%), DEN-1 (11 \%) y DEN-4 (7,8 \%). La abundancia de cada serotipo varió en el tiempo (Figura 1). El DEN-1 se aisló más entre 1998-999 (10 de 14 cepas); el DEN-2 fue permanente y la mayoría de aislados fueron entre 1998-2001 (25 de 29 cepas); el DEN-3 no se aisló entre 19982000, reapareció en el 2001 luego de 22 años ausente de Colombia y en los años siguientes (2002-2004) se hizo prevalente (67 de 76 cepas); el DEN-4 se aisló en 2000-2001 (7 de 10 cepas) y de nuevo en el 2004.

DH, serotipo dominante y tipo de infección

Cambios temporales de la frecuencia del DH coincidieron con cambios de predominancia de serotipos y frecuencia de infecciones secundarias (Tabla 1). Entre 2000-2001 cuando hubo más DH (> 7 \%) el DEN-2 fue predominante (18 de 26 virus aislados) y esto coincidió con más infecciones secundarias (>73 \%). Entre 2002-2004 menos casos de DH ocurrieron junto con más infecciones primarias ( $>80 \%$ ) y más dominancia del DEN-3 (66 de 75 virus). Entre 2000-2004 hubo disminución anual del DH que correlacionó con menos DEN-2 ( $\mathrm{r}=0,95 ; \mathrm{p}=0,01)$, menos infecciones secundarias $(\mathrm{r}=0,9 ; \mathrm{p}=0,03)$ e incremento del DEN-3 ( $r=-0,91 ; \mathrm{p}=0,03)$. La dominancia del DEN-1 coincidió con el brote de 1998 cuando la proporción de casos hemorrágicos fue baja (3,7 \%) y la circulación del DEN-4 no pareció contribuir de manera significativa al DH.

Severidad del dengue en casos con aislamiento del virus

En 146 casos de quienes el virus se aisló la información clínica y de laboratorio fue completa y confiable. La edad (años) fue $23 \pm 23,25 \pm 18,26 \pm 16$ y $27 \pm 14$, en quienes se aisló el DEN-1,2,3 y 4, respectivamente. De los infectados con DEN-2 ( $n=53$ ) 24,5 \% desarrollaron DH (tres fatales) mientras $11,2 \%$ de con DEN-3 $(n=71)\left(p<0,05, \chi^{2}\right)$. Por otro lado, $56,8 \%$ de las infecciones por DEN2 fueron secundarias mientras que $15,7 \%$ de las de DEN-3 $\left(p<0,001, \chi^{2}\right)$. De los tres casos fatales por DEN-2 dos tuvieron infección secundaria, de los 
infectados con DEN-1, 4 (23 \%) sufrieron DH y todos los casos por DEN-4 $(\mathrm{n}=9)$ fueron DC (Figura 2). En total, 25 (17,1 \%) casos de los que tuvieron aislamiento del virus desarrollaron $\mathrm{DH}$.

Figura 1. Frecuencia anual de serotipos del virus dengue, aislados en el Departamento de Santander en el período 1998 - 2004.

口DEN-1 $\square$ DEN-2 $\square D E N-3$ aDEN-4

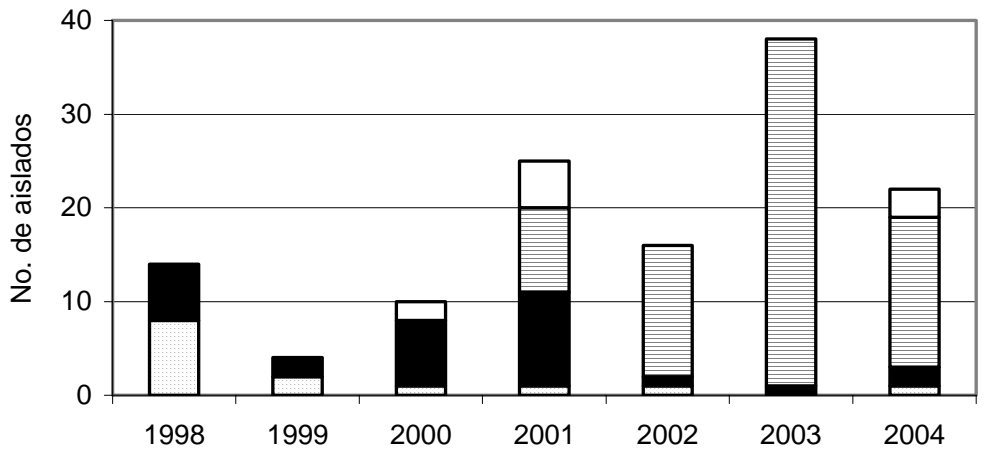

Tabla 1. Relación entre frecuencia anual del $\mathrm{DH}$, casos de infección secundaria y serotipo dominante. Departamento de Santander, 1998 - 2004.

\begin{tabular}{|c|c|c|c|c|c|c|c|c|}
\hline \multirow[b]{2}{*}{ Año } & \multicolumn{2}{|c|}{ Casos de $\mathrm{DH}^{\top}$} & \multicolumn{2}{|c|}{ Infección $2^{\mathrm{a}}$. } & \multicolumn{4}{|c|}{ Serotipo: dominancia } \\
\hline & No. & $\%$ & $+/ \mathrm{No}$ & $\%$. & D1 & D2 & D3 & D4 \\
\hline 1998 & 881 & 3,7 & $69 / 80$ & 86,3 & 57,1 & 42,9 & 0 & 0 \\
\hline 2000 & 130 & 8,6 & $63 / 84$ & 75,0 & 10 & 70 & 0 & 20 \\
\hline 2001 & 779 & 7,4 & $94 / 128$ & 73,5 & 4 & 40 & 36 & 20 \\
\hline 2002 & 520 & 4,7 & $58 / 106$ & 54,8 & 6,7 & 6,7 & 86,6 & 0 \\
\hline 2003 & 285 & 4,3 & $18 / 80$ & 22,5 & 0 & 2,7 & 97,3 & 0 \\
\hline 2004 & 58 & 3,5 & $22 / 118$ & 18,6 & 4,8 & 9,5 & 71,4 & 14,3 \\
\hline
\end{tabular}

${ }^{\dagger}$ : Ref.18. *: número de aislamientos del serotipo/total de virus x 100. Disminución del DH entre 20002004 correlacionó con disminución de la dominancia del DEN-2 $(r=0,95 ; p=0,01)$ y de la infección secundaria $(r=0,9 ; p=0.03)$ e incremento de la dominancia del DEN-3 $(r=-0,91 ; p=0,03)$

Respuesta de anticuerpos neutralizantes y DH

Se investigó en casos secundarios de DH de 1998 (DH 3,7 \%) y 2001 (DH 7,4 $\%$ ), en un intento de establecer la relación entre carga inmune al dengue y severidad (Tabla 2). Se detectaron títulos mayores de anticuerpos anti-DEN1,3 y 4 en adultos y niños del 2001 (DEN-2 y 3 dominantes) que de 1998 (DEN-1 dominante) y al contrario con anticuerpos contra DEN-2, aunque en ningún caso las diferencias fueron significantes ( $p>0,05$, prueba de Mann- 
Whitney $U$ ). El título de los anticuerpos se determinó en 15 pacientes (6 de DH) con infección secundaria por DEN-2, detectándose en 14 respuesta heterotípica, es decir, anticuerpos neutralizantes de los cuatro serotipos a título $\geq 1: 340$ (Tabla 3). El otro caso (fatal) fue un niño de 6 meses con anticuerpos contra el virus aislado y contra el DEN-1 solamente, indicando que el niño sufrió una infección primaria por DEN-2 y que los anticuerpos anti-DEN-1 pudieron ser trasmitidos por la madre. Títulos de anticuerpos contra el virus de la infección actual (DEN-2) $\geq$ 1:10 000 fueron más frecuentes en casos de DC que de DH: $77,7 \%$ vs 33,3\%, lo mismo que títulos mayores de anticuerpos ( $\geq 1: 1200$ ) contra los otros serotipos. Los resultados sugieren que la magnitud de la respuesta de anticuerpos podría ser un factor determinante de la severidad del dengue: cuanto menor más probabilidad de DH.

Figura 2. Severidad y tipo de infección en casos de dengue en relación con el serotipo del virus aislado

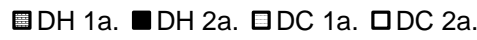

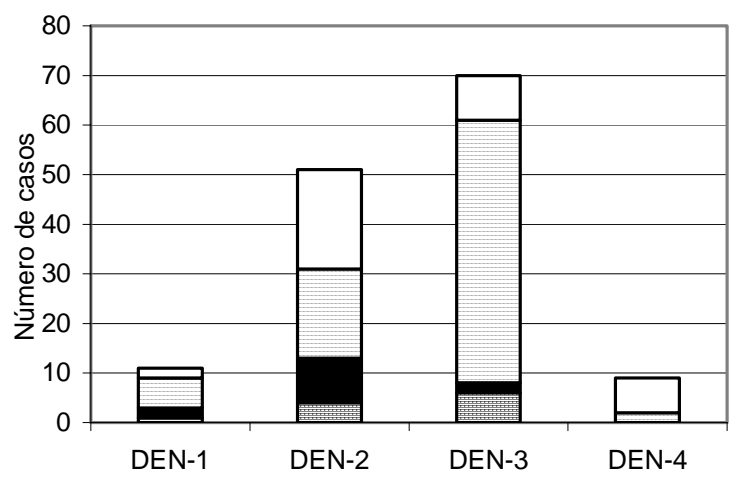

DH 1a: dengue hemorrágico por infección primaria. DH 2a: dengue hemorrágico por infección secundaria DC 1a: dengue clásico por infección primaria. DC 2a: dengue clásico por infección secundaria

Tabla 2. Título de anticuerpos neutralizantes contra serotipos del virus en casos de dengue de años con diferencia frecuencia de $\mathrm{DH}^{\dagger}$. Departamento de Santander.

\begin{tabular}{|c|c|c|c|c|c|c|}
\hline \multirow[b]{2}{*}{ Virus } & \multicolumn{3}{|c|}{ 1998: $\mathrm{DH} 3,4 \%^{\mp}$} & \multicolumn{3}{|c|}{ 2001: DH 7,4 \%\& } \\
\hline & $\begin{array}{c}0,6-15^{n} \\
n=17\end{array}$ & $\begin{array}{c}>15^{n} \\
n=39\end{array}$ & Prom.* & $\begin{array}{c}0,6-15 \\
n=14\end{array}$ & $\begin{array}{c}>15 \\
n=41\end{array}$ & Prom. \\
\hline DEN-1 & 3519 & 7004 & 5262 & 6193 & 8138 & 7166 \\
\hline DEN-2 & 5818 & 5933 & 5876 & 3639 & 6117 & 4878 \\
\hline DEN-3 & 1501 & 3767 & 2634 & 3681 & 5456 & 4569 \\
\hline DEN-4 & 771 & 2534 & 1623 & 2371 & 2816 & 2594 \\
\hline
\end{tabular}

†:Ref.18. ‡: DEN-1 dominante. \&: DEN-2 y 3 dominantes. ๆ: edad en años. *: promedio. Los datos corresponden al promedio del recíproco de la mayor dilución del suero que neutralizó el virus (PRNT ${ }_{80}$ ). No hubo diferencias cuando se compararon entre sí los títulos para cada no de los virus de cada grupo de edad de cada año $(P>0,05$, prueba de Mann-Whitney $U$.) 


\section{DISCUSIÓN}

El incremento significativo del DH/SCH en América desde los años 80 ha sido ampliamente atribuido a la circulación simultánea de los cuatro serotipos, sumado a la introducción de la cepa asiática (subtipo III) del DEN-2 e indiana (subtipo III) del DEN-3 $(14,15)$. El primer virus causó las muertes en las epidemias cubanas de 1981 y $1997(12,22)$ y es el que más se aisla de casos hemorrágicos en todos los países $(5,6,9)$. El segundo se ha relacionado con el incremento del DH en la isla de Sri-Lanka desde 1986 (13,23). En Colombia los cuatro serotipos están circulando hace más de 20 años y el genotipo ha sido documentado para todos menos el 3. Virus DEN-2 de 1989 y 1996 resultaron genotipo III, mientras que aislados en años anteriores (1986 y 1988) fueron I o americano (10) y virus DEN-1 (1985 y 1996) y DEN-4 (1985 y 1996) se agruparon en los genotipos III y II, respectivamente $(24,25)$. Los resultados de este estudio demostraron que los cuatro virus del dengue circularon en el departamento de Santander entre 1998-2004, exhibiendo dominancia anual que coincidió con cambios en la incidencia del dengue y de casos hemorrágicos. Algunas de las cepas aisladas fueron tipificadas en nuestro laboratorio usando la técnica de amplificación simultánea de secuencias para enzimas de restricción o RSSPCR (26). Virus de los serotipos 2 y 3 resultaron subtipo Ay C, respectivamente, los cuales corresponden a virus genotipo III de cada serotipo, en tanto que virus serotipo 4 fueron subtipo B (genotipo II) el único detectado en América (27).

Tabla 3. Título de anticuerpos neutralizantes de serotipos del virus en casos con infección secundaria por DEN-2. Departamento de Santander 1998 y 2001

\begin{tabular}{|c|c|c|c|c|c|}
\hline $\begin{array}{l}\text { Edad } \\
\text { (años) }\end{array}$ & D1 & D2 & D3 & D4 & Severidad* \\
\hline 4 & 640 & 10240 & 2560 & 2560 & $\mathrm{DC}$ \\
\hline 5 & 640 & 10240 & 640 & 2560 & DC \\
\hline 10 & 640 & 10240 & 2560 & 2560 & DC \\
\hline 16 & 2560 & 10240 & 2560 & 340 & DC \\
\hline 20 & 640 & 640 & 640 & 340 & DC \\
\hline 25 & 2560 & 10240 & 2560 & 640 & DC \\
\hline 29 & 1280 & 2560 & 1280 & 1280 & DC \\
\hline 32 & 10240 & 10240 & 2560 & 340 & DC \\
\hline 43 & 340 & 10240 & 2560 & 2560 & DC \\
\hline 0,6 & 640 & 640 & Neg & Neg & $\mathrm{DH} /$ fatal \\
\hline 23 & 640 & 640 & 340 & 340 & $\mathrm{DH}$ \\
\hline 29 & 640 & 640 & 340 & 340 & $\mathrm{DH}$ \\
\hline 46 & 640 & 10240 & 2560 & 640 & $\mathrm{DH}$ \\
\hline 73 & 640 & 640 & 340 & 340 & $\mathrm{DH} /$ fatal \\
\hline 46 & 2560 & 10240 & 640 & 340 & $\mathrm{DH}$ \\
\hline
\end{tabular}


El DEN-2 fue el serotipo más aislado de los casos de DH identificados en este estudio y fue el virus dominante en los años con más infecciones secundarias y más casos severos (Tabla 1). Varios autores han confirmado la asociación entre infección secundaria por el DEN-2 subtipo III y DH (5,6,9,12). El riesgo es mayor en infectados previamente con DEN-1 (OR=502,7), menos con DEN-3 $(\mathrm{OR}=157,4)$ y aún menos con DEN-4 $(\mathrm{OR}=57,5)(28)$. En el Departamento de Santander el DEN-1 dominó entre 1997-1999 cuando se reportaron cerca de 24000 casos de dengue (18), pero en los 5 años siguientes los aislamientos de este serotipo fueron muy escasos (Figura 1). En ese contexto, se podría asumir que en 2000-2001 cuando más DH hubo, había una proporción significante de individuos con anticuerpos previos anti-DEN-1 que pudieron infectarse con DEN-2 (dominante). Dado el diseño del estudio no pudo concluirse sobre la asociación entre frecuencia del DH y anticuerpos de infecciones pasadas. Sin embargo, el título de anticuerpos neutralizantes en casos del 2001 fue mayor para DEN-1 que para los serotipos dominantes (2 y 3) y no hubo diferencias con los casos del año 1998 cuando este virus dominó (Tabla 2). Este hallazgo sugiere investigar si los pobladores de Santander portan mayor carga inmune al DEN-1 que a los otros virus o si los anticuerpos anti-DEN-1 tienen mayor reactividad cruzada. Si esto es así, se esperaría ocurrencia de epidemias con más casos hemorrágicos años después de un brote por DEN-1 cuando el DEN-2 sea el predominante. Estudios epidemiológicos son necesarios para estudiar este aspecto como factor de riesgo de DH en el Departamento.

El DEN-3 causó más casos leves y su dominancia coincidió con menos DH (Tabla 1). Lo mismo se observó en Puerto Rico (29), México (30) y Venezuela (31) siguiendo la re-introducción del virus. En el año de su reaparición en esos países la mayoría de los casos severos fueron por DEN-2 y algunos por DEN-1. Igualmente, la dominancia del virus en Nicaragua en 1998 produjo una epidemia pero el DEN-2 fue el más aislado de casos severos (32). No obstante, la introducción del DEN-3 por primera vez en Brasil en 2000 resultó en la epidemia con más casos severos registrada en ese país (33). Las causas de la mayor severidad vista en Brasil no han sido aún esclarecidas pero podrían estar relacionadas con la susceptibilidad genética y carga inmune de la población junto con mayor virulencia de la cepa.

Los resultados presentados en este reporte soportan el concepto que el riesgo de epidemias de DH está relacionado con cambios en la dominancia de los serotipos del virus y ésta con cambios en la carga inmune de la población. Como consecuencia, la vigilancia virológica continua y 
permanente deberá ser una prioridad de los programas de control en las áreas colombianas donde el dengue es endémico

Agradecimientos. Al grupo de epidemiología y del Laboratorio de Salud Pública, Secretaría de Salud de Santander, por el soporte logístico. Al Dr. Vancen Vorndam (CDC, Puerto Rico) por el suministro de antígeno y anticuerpos monoclonales, a los Drs. Luis Angel Villar, Ruth Martínez y Fredi Díaz por la información clínica de los pacientes. Apoyo financiero: COLCIENCIAS (Proyecto No ${ }^{\circ} 11020413042$ ) y Secretaría de Salud del Departamento de Santander (Contrato No 133-99).

\section{REFERENCIAS}

1. Henchal E, Putnak R. The Dengue viruses. Clin Microbiol Rev. 1990; 3:376-396.

2. Lanciotti RS, Lewis JL. Gubler DJ, Trent DW. Molecular evolution and epidemiology of dengue-3 viruses. J Gen Virol. 1994; 75: 65-75.

3. Lanciotti RS, Gubler DJ, Trent DW. Molecular evolution and phylogeny of dengue4 viruses. J Gen Virol 1997;78:2279-2284.

4. Rico-Hesse R. Molecular evolution and distribution of dengue viruses type 1 and 2 in nature. Virology 1990;174:479-493.

5. Vaughn DW, Green S, Kalajanarooj S, Innis BL, Nimmannitya S, Suntayakorns S, et al. Dengue viremia titer, antibody, response pattern and virus serotype correlate with disease severity. J Infect Dis. 2000; 181:2-9.

6. Burke DS, Nisalak A, Johnson D, Scott R. A prospective study of dengue infections in Bangkok. Am J Trop Med Hyg. 1988; 38:172-180.

7. Kurane I, Ennis FA. Immunopathogenesis of dengue viruses infections. In: Gubler DJ, Kuno G (Ed). Dengue and dengue hemorrhagic fever. London: CAB International. 1997. p. 273-290.

8. Halstead SB. Neutralization and antibody-dependent enhancement of dengue viruses. Adv Virus Res. 2003; 60:421-467.

9.Nisalak A, Endy TP, Nimmannitya S, Endy TP, Nimmannitya S, Kalayanarooj S, et al. Serotype-specific dengue virus circulation and dengue disease in Bangkok, Thailand from 1973 to 1999. Am J Trop Med Hyg. 2003;68:191-202.

10. Rico-Hesse R, Harrison L, Salas R, Tovar D, Nisalak A, Ramos C, et al. Origins of dengue type 2 viruses associated with increased pathogenicity in the Americas. Virology 1997; 230:244-251.

11. Pinheiro FP, Corber SJ. Global situation of dengue and dengue haemorrhagic fever, and its emergence in the Americas. World Health Stat Q.1997; 50, 161-169.

12. Kouri GP, Guzman MG Bravo JR. Why dengue hemorrhagic fever in Cuba? 2. An integral analysis. Trans R Soc Trop Med Hyg. 1987; 81:821-823. 
13. Messer WB, Gubler DJ, Harris E, Sivananthan K, de Silva AM. Emergence and global spread of a dengue serotype 3, subtype III virus. Emerg Infect Dis. 2003;9:800-809.

14. Guzmán MG, Kouri G. Dengue and dengue hemorrhagic in the Americas. lessons and challenges J Clin Virol. 2003; 27:1-3.

15. Gubler JD, Clark CG. Dengue/Dengue hemorrhagic fever: the emergence of a global health problem. Emerg Infect Dis. 1995; 1: 55-57.

16. Boshell J, Groot H, Gacharna M, Márquez G, González M, Gaitán MO, et al. Dengue en Colombia. Biomédica 1986; 6: 101-102.

17. PAHO (2000-2004). Number of Reported Cases of Dengue, Dengue Hemorrhagic Fever (DHF). Region of the Americas (by country and subregion). [Internet]. Disponible en: http://www.paho.org/english/ad/dpc/cd/dengue.htm Consultado en: Marzo 22 de 2007.

18. Álvarez A, Martínez H, Millán S, López L. Diagnóstico de la situación de Salud en Santander. 2. Ed. Secretaría de Salud de Santander; 2004 p. 56-60.

19. World Health Organization. Guide for diagnosis, treatment and control of dengue hemorrhagic fever. Technical Advisory Committee on Dengue hemorrhagic fever for the South East Asian and Western Pacific regions. Geneva: World Health Organization; 1980.

20. Ocazionez R, Cortés F, Villar LA. Vigilancia del dengue basada en el laboratorio: diferencias en el número de casos y virus aislados según la recolección del suero y la prueba serológica. Colombia Médica 2005; 36: 65-72.

21. Vorndam V, Beltran M. Enzyme-linked immunosorbent assay-format microneutralization test for dengue viruses. Am J Trop Med Hyg. 2002; 66: 208-212.

22. Rodríguez-Roche R, Alvarez M, Gritsun T, Halstead S, Kouri G, Gould EA, et al. Virus evolution during a sever dengue epidemic in Cuba, 1997. Virology 2005;334:154159.

23. Messer BW, Vitarana UT, Sivananthan K, Vigala JE, Preethimala LD, Ramesh R, et al. Epidemiology of dengue in Sri-Lanka before and after of emergence of epidemic dengue hemorrhagic fever. Am J Trop Med Hyg. 2002; 66: 765-773.

24. Gonçalvez A, Escalante A, Pujol FH, Ludert J, Tovar D, Salas R, et al. Diversity an evolution of the envelope gene of Dengue virus type 1. Virology 2002: 303; 110-119.

25. Diaz FJ, Black W, Farfán-Ale J, Loroño-Pino M, Olson K, Beaty B. Dengue virus circulation an evolution in Mexico: A phylogenetic perspective. Arch Med Res. 2006;760-773.

26. Harris E, Sandoval E, Xet-Mull AM, Johnson M, Riley LW. Rapid subtyping of dengue viruses by restriction site-specific (RSS)-PCR. Virology 1999; 253: 8695. 
27. Cortés FM, Gómez SY, Ocazionez RE. Subtipos de virus dengue serotipos 2, 3 y 4 aislados en el Departamento de Santander, Colombia. Rev Cubana de Medicina Tropical 2007, en preparación.

28. Endy TP, Nisalak A, Chunsuttitwat S, Vaugh DW, Green S, Ennis FA, et al. Relationship of preexisting dengue virus (DV) neutralizing antibodies levels to viremia and severity desease in a prospective cohort study of DV infection in Thailand. J Infect Dis. 2004; 189: 990-1000

29. Rigau JC, Ayala A, García E, Hudson SM, Vordam V, Reiter P, et al. The reappearance of dengue- 3 and a subsequent dengue- 4 and dengue- 1 epidemic in Puerto Rico. Am J Trop Med Hyg. 2002; 67: 355-362.

30. Briceño-García B, Gómez-Dantés H, Argot-Ramírez E, Montesano R, VázquezMartínez AL, Ibañez-Bernal S, et al. Potential risk for dengue hemorrhagic fever: The Isolation of serotype Dengue-3 in Mexico. Emerg Infect Dis. 1996; 2:133-135.

31. Uzcategui NY, Comach G, Camacho D, Salcedo M, Cabello de Quintana M, Jimenez $\mathrm{M}$, et al. Molecular epidemiology of dengue virus type 3 in Venezuela. J Gen Virol. 2003; 84:1569-75.

32. Harris E, Videa E, Perez L, Sandoval E, Tellez Y, Perez ML, et al. Clinical, epidemiologic, and virologic features of dengue in the 1998 epidemic in Nicaragua. J Trop Med Hyg. 2000; 2:5-11.

33. Nogueira RM, Schatzmayr HG Filippis AMB, Santos FV, Cunha RV, Coehlo JO, et al. Dengue Virus Type 3, Brazil, 2002. Emerg Infect Dis 2005; 11: 1376-1381. 